\title{
A GESTÃO SOCIOAMBIENTAL À LUZ DAS TÉCNICAS DE VALORACÃO ECONOMICA DO MEIO AMBIENTE: UMA ANÁLISE DO VALOR DE USO INDIRETO E DO VALOR DE EXISTÊNCIA
}

\author{
SOCIOENVIRONMENTAL MANAGEMENT IN LIGHT OF TECHNIQUES FOR ECONOMIC VALUATION \\ OF THE ENVIRONMENT: AN ANALYSIS OF THE VALUE OF INDIRECT USE AND THE VALUE OF \\ EXISTENCE
}

LA GESTIÓN SOCIOAMBIENTAL A LA LUZ DE LAS TÉCNICAS DE VALORACIÓN ECONÓMICA DEL MEDIO AMBIENTE: UN ANÁLISIS DEL VALOR DE USO INDIRECTO Y DEL VALOR DE EXISTENCIA

Glaucia de Paula Falco

Doutor

Faculdade Machado Sobrinho glaupf@terra.com.br

Marley Maria Bernardes Rebuzzi Vellasco

Doutor

Pontifícia Universidade Católica do Rio de Janeiro marley@ele.puc-rio.br

Juan Guilhermo Lazo Lazo juan@ele.puc-rio.br

Joyce Gonçalves Altaf

Mestre

Fundação Educional Machado Sobrinho

jgaltaf@yahoo.com.br

Irene Raguenet Troccoli

Doutor

Universidade Estácio de Sá irene.troccoli@estacio.br

Submissão em: 17/03/2011

Aprovado em: 30/08/2012

RESUMO

Atualmente, o meio ambiente é uma das maiores preocupações da sociedade mundial. Contudo identificar e monitorar os problemas ambientais e a forma como estes interferem na qualidade de vida dos indivíduos é uma questão muito abstrata: não existe uma "receita de bolo" para inserir a gestão econômica na gestão ambiental e nas decisões de investimento. O objetivo deste artigo é desenvolver uma nova metodologia para valoração do meio ambiente a partir dos conceitos base da teoria de opções reais que evite a subestimação do valor dos recursos naturais, ao permitir explicitamente calcular as parcelas do valor de uso indireto, do valor de existência e valor de quasi opção como componentes do valor total do meio ambiente. A proposta deste desenvolvimento é relevante porquanto uma das vantagens dos métodos de valoração é permitir internalizar os custos ambientais decorrentes da atividade econômica. Assim, a partir de um valor monetário, é possível adotar políticas específicas para proteger o meio ambiente, tais 
como taxação, aumento de impostos, multas e indenizações. Ou até mesmo adiar a decisão de degradar a natureza - ação que, sob certas condições, pode ser a melhor escolha.

PALAVRAS-CHAVE: Gestão socioambiental. Valoração. Valor de uso indireto. Valor de existência.

\begin{abstract}
The environment is one of the major concerns of society worldwide. However, identifying and monitoring environmental problems, and how they affect the quality of life of individuals, are very abstract issues. There is no "magic formula" for including economic management in environmental management and investment decisions. The aim of this paper is to develop a methodology for valuing the environment based on the theory of real options that avoids the underestimation of the value of natural resources, for explicitly calculating the portions of value of indirect use, the value of existence and the value of quasi option, as components of the total value of the environment. The purpose of this development is relevant because one of the advantages of the valuation methods is to internalize the environmental costs associated with economic activity. Thus, based on a monetary value, it is possible to adopt specific policies for the protection of the environment, such as taxation, increased taxes, fines and compensations, or even to postpone the decision to degrade nature - an action that, under certain conditions, may be the best choice.
\end{abstract}

KEYWORDS: Environmental management. Valuation. Value of indirect use. Value of existence.

\title{
RESUMEN
}

Actualmente, el medio ambiente es una de las mayores preocupaciones de la sociedad mundial. Sin embargo, identificar y monitorear los problemas ambientales y la forma en que estos interfieren en la calidad de vida de los individuos es una cuestión muy abstracta: no existe una "fórmula lista para usar" para insertar la gestión económica en la gestión ambiental y en las decisiones de inversión. El objetivo de este artículo es desarrollar una nueva metodología para la valoración del medio ambiente a partir de los conceptos base de la teoría de opciones reales, que evite la subestimación del valor de los recursos naturales al permitir, explícitamente, calcular los porcentajes del valor de uso indirecto, del valor de existencia y valor de cuasi opción como componentes del valor total del medio ambiente. La propuesta de este desarrollo es relevante puesto que una de las ventajas de los métodos de valoración es permitir la internalización de los costos ambientales decurrentes de la actividad económica. Así, a partir de un valor monetario, es posible adoptar políticas específicas para proteger el medio ambiente, tales como tributación, aumento de impuestos, multas e indemnizaciones. $\mathrm{O}$, inclusive, posponer la decisión de degradar la naturaleza - acción que, bajo ciertas condiciones, puede ser la mejor elección.

PALABRAS CLAVE: Gestión socioambiental. Valoración. Valor de uso indirecto. Valor de existencia.

\section{INTRODUÇÃO}

De acordo com Faria e Silva (1999), a gestão eficiente dos recursos ambientais implica reconhecerem-se as oportunidades de investimentos oriundas da gestão ambiental. Ao se falar em gestão econômica eficiente dos recursos naturais, indiretamente aparece a necessidade de se conhecer o valor econômico do meio ambiente. Para o investidor ou empresário, quando a tomada de decisão envolve o meio ambiente a análise de investimento passa pela tradicional análise custobenefício, que é muito mais bem compreendida quando pode ser expressa em valores monetários. Isso permite comparar distintas opções. 
A necessidade de conhecer o valor econômico dos bens ambientais e incorporá-los ao processo econômico é uma preocupação antiga, mas se intensificou nos últimos 30 anos. O motivo da crescente preocupação com a natureza se deve à conscientização de que os recursos naturais, antes considerados ilimitados e de domínio público, na realidade são escassos. Os recursos naturais - ou capital natural - sempre foram tratados, pela Humanidade, como uma fonte inesgotável de suprimento de matéria-prima. Mas isso mudou nas últimas décadas (FREEMAN, 1979; MOTA, 2006; BENAKOUCHE; CRUZ, 1994; PEARCE; TURNER, 1990).

Atualmente, o meio ambiente é uma das maiores preocupações da sociedade mundial. A questão ambiental ocupa a terceira posição no ranking das questões em foco desta geração. Entre outros motivos, isso se deve aos efeitos negativos do padrão de consumo, que tem afetado não apenas as condições físicas do ar, da terra e da água, mas também a saúde das pessoas e o ambiente social construído pelo homem para manter a sua existência na terra (SOUZA, 2007; OLIVEIRA, 2005).

É compreensível que, à medida que os indivíduos se conscientizem dos danos da degradação ambiental e de suas consequências para o planeta e à vida humana, aumente a mobilização, nas sociedades, pela proteção dos recursos naturais. Portanto quanto mais as pessoas se tornam esclarecidas quanto às preocupações ambientais - como, por exemplo, o buraco na camada de ozônio, o desmatamento de florestas, as chuvas ácidas e a poluição -, tanto mais os aspectos ambientais farão parte das suas decisões de consumo, de produção e de preservação.

Contudo identificar e monitorar os problemas ambientais e a forma como estes interferem na qualidade de vida dos indivíduos é uma questão muito abstrata (EUSTÁCHIO; TÁVORA, 1999). Isso porque, como bem destaca Motta (1997), não existe uma "receita de bolo" para inserir a gestão econômica na gestão ambiental e nas decisões de investimento. Uma tentativa de conciliar os interesses econômicos com os interesses da conservação ambiental é proposta por meio da valoração monetária dos recursos naturais, mas essa não tem sido uma tarefa fácil.

Considerando a decisão de uso dos recursos naturais, isso envolve ainda decidir sobre quanto usar dos recursos e como usá-los. Neste sentido, é válido destacar que cabe à geração atual cuidar para que as futuras gerações tenham quantidade suficiente de bens ambientais para satisfazerem suas necessidades. Além disso, como é preciso garantir a continuidade do desenvolvimento sustentável, isto significa também pensar em como esta geração e as próximas gerações conduzirão o intercâmbio natureza-homem-atividade econômica (BRAGA; OLIVEIRA; ABDALLAH, 2008; MOTA, 2006).

Para a Comissão Mundial de desenvolvimento e meio ambiente, o conceito de desenvolvimento sustentável relaciona-se com a trajetória do progresso humano condicionado pelas limitações tecnológicas, a organização social do planeta e a capacidade da biosfera assimilar os efeitos da atividade econômica e humana (MOTA, 2006; MOTTA, 1997a).

Conforme o estudo intitulado de "Nosso futuro comum" de 1991, da Comissão Mundial de Meio Ambiente e Desenvolvimento, o desenvolvimento sustentável pode ser definido como "aquele que atende às necessidades do presente sem comprometer a possibilidade de as gerações futuras atenderem as suas próprias necessidades" (MOTA, 2006, p. 23). Observe que o desenvolvimento sustentável aparece estreitamente ligado ao monitoramento da quantidade de recursos naturais disponíveis.

No entanto há uma série de problemas na alocação eficiente e na administração da utilização dos recursos naturais. Um deles envolve a escolha da alocação dos recursos entre os vários usos competitivos, visando maximizar o bem-estar e a satisfação da sociedade (AUSTRALIAN GOVERNANMENT PUBLISHING SERVICE, 1995). Como escolher qual é o melhor uso?

Por exemplo, um parque tanto pode ser usado para fins recreativos quanto para o corte de árvores e, posteriormente, para a venda da madeira (CONRAD, 1999; INSLEY, 2002). Neste caso, o debate deverá levar em conta os benefícios e os custos tanto da preservação quanto da destruição trazida pelo corte para a venda da madeira. Esta discussão aparece no trabalho de Pindyck (1999), que avalia o custo-benefício de se adotar uma política ambiental para reduzir as quantidades de $\mathrm{CO}_{2}$ emitidas para a atmosfera.

Outro problema na alocação eficiente dos ativos naturais se relaciona com os benefícios e os custos futuros associados a uma determinada alternativa de uso, levando em conta o aspecto intertemporal da utilização dos recursos. Portanto se observa que não basta computar os valores 
presentes dos benefícios e dos custos: é preciso tentar mensurar as consequências, para as futuras gerações, associadas a uma decisão tomada no instante presente. Pearce e Turner (1990) também associam o desenvolvimento sustentável com a manutenção dos benefícios líquidos do desenvolvimento econômico e com a qualidade dos recursos naturais ao longo do tempo. A esse respeito, diz Motta (2000, p.1) que "O uso dos recursos naturais gera custos externos negativos intra e intertemporais".

Desta forma, evidencia-se relação estreita, com a proposta de avaliação monetária do meio ambiente, do uso racional dos recursos naturais e dos conceitos de desenvolvimento sustentável e de gestão socioambiental - foco deste trabalho. Conforme destacado por Nogueira, Medeiros e Arruda (2000), quaisquer medidas ou ações gerenciais que busquem o desenvolvimento e o uso sustentável dos ativos naturais se beneficiarão da estimação de valores monetários para estes ativos.

Como os recursos naturais não são transacionados nos mercados convencionais, é preciso encontrar mecanismos que possibilitem internalizar ao sistema econômico de trocas os custos e os benefícios associados à utilização dos bens ambientais (SOUZA, 2007; SOUZA; SOUZA, 2008). Neste momento, surge a necessidade de valorar o meio ambiente, e as técnicas de avaliação dos recursos naturais ganham grande importância.

Sendo assim, o objetivo deste artigo é desenvolver uma metodologia para valoração do meio ambiente a partir dos conceitos da teoria de opções reais que evite a subestimação do valor dos recursos naturais ao permitir explicitamente calcular as parcelas do valor de uso indireto, valor de existência e valor de quasi opção como componentes do valor total do meio ambiente. O racional desta proposta reside no fato de essas parcelas comporem este valor total, o que implica que ignorá-las significa subestimar o valor dos recursos naturais, podendo gerar distorções no processo de tomada de decisão. Outrossim, observe-se que o termo meio ambiente aqui estará referido aos recursos naturais disponíveis, que não são produzíveis pelo homem e que geram benefícios econômicos, sociais e funcionais.

\section{REFERÊNCIAL TEÓRICO}

O tema ambiental é bastante amplo e sua definição envolve uma gama de conceitos que passa por diversas áreas de conhecimento, tais como economia, biologia, filosofia, geologia, direito, sociologia, entre outras. Cada uma destas disciplinas desenvolveu o seu conjunto de questões, teorias e métodos para abordá-lo segundo os seus objetivos. Atualmente o meio ambiente é uma das maiores preocupações desta geração (MOTA, 2006).

Como bem pondera Facin (2002), nas palavras de Vladimir Passos de Freitas em "Direito Administrativo e Meio Ambiente" (2002, p. 7):

\footnotetext{
O meio ambiente é, atualmente, um dos poucos assuntos que desperta o interesse de todas as nações, independentemente do regime político ou sistema econômico. É que as conseqüências dos danos ambientais não se confinam mais nos limites de determinados países ou regiões. Ultrapassam as fronteiras e, costumeiramente, vêm a atingir regiões distantes. Daí a preocupação geral no trato da matéria que, em última análise, significa zelar pela própria sobrevivência do homem.
}

Por definição, meio ambiente é o local em que as pessoas vivem e expandem a vida. Portanto o meio ambiente é um direito econômico de todos. Por isso é importante preservá-lo e compatibilizar a conservação com o desenvolvimento econômico e social, respeitando o direito das futuras gerações usufruirem de um ambiente dotado de qualidade. Esta é uma razão pela qual o assunto "meio ambiente" tem assumido papel de destaque nas Constituições de todos os países (FACIN, 2002). Na pesquisa desenvolvida pelo presente trabalho, o termo "meio ambiente" está se referindo aos recursos naturais disponíveis, que não são produzíveis pelo homem e que geram benefícios econômicos, sociais e funcionais.

Os benefícios funcionais do ambiente se referem à função ecossistêmica destes bens naturais no equilíbrio das condições de vida no planeta. Sabe-se que os ecossistemas estão em constante interação com o meio ambiente, com os processos químicos e com as leis ecológicas e econômicas (MOTA, 2006). Assim, a função ecossistêmica do ambiente possibilita a manutenção das condições microclimáticas favoráveis à existência humana, à existência da fauna e da flora, e à saúde física e 
mental da população que vive próxima das áreas verdes, além de minimizar os efeitos da poluição do ar (HILDERBRAND; GRAÇA; HOEFLICH, 2002). Isto permite o equilíbrio natural necessário à vida.

\section{A valoração econômica como instrumento para a gestão socioambiental dos recursos naturais}

Valorar o meio ambiente significa atribuir um valor econômico para os recursos naturais e seu entorno, entendendo-se este valor como aquele que reflete a maior ou a menor importância que as pessoas atribuem a um determinado bem, dependendo das condições (MANKIW, 2008).

Observe que este valor, apesar de possuir uma expressão monetária, não deve ser entendido como o "preço" ${ }^{1}$ do recurso, pois o meio ambiente possui um valor inestimável (FREEMAN, 1979; BENAKOUCHE; CRUZ, 1994). O sentido de atribuir valor numérico é criar um indicativo do bem-estar proporcionado pelo bem ambiental, que sirva como um denominador comum entre o meio ambiente e os demais bens e serviços. Isso permitirá fazer comparações que poderão subsidiar as decisões a serem tomadas na gestão dos recursos naturais (HILDERBRAND; GRAÇA; HOEFLICH, 2002; MATTOS, 2006), considerando-se que, nos mercados, os bens são racionados pelos preços (MANKIW, 2008).

Apesar da importância reconhecida de se ter um valor numérico associado aos recursos naturais, identificar, qualificar e quantificar os danos ambientais decorrentes de uma ação que interfere no equilíbrio natural envolve muitos aspectos sobre os quais ainda não há um consenso. Um exemplo destes aspectos é o valor da vida: qual é ele? A resposta para esta pergunta abrange uma série de fatores difíceis de serem explicitados e quantificados, dada a inexistência de um mercado em que a vida possa ser comercializada.

Assim, o que valorar? Essa pergunta deve ser respondida segundo uma prioridade previamente definida, considerando que, apesar dos mecanismos de mercado não capturarem os valores dos ativos ambientais, existe um valor econômico associado aos recursos naturais (MOTTA, 1997; BENAKOUCHE; CRUZ, 1994). Isso porque o seu uso provoca impactos no processo de produção e de consumo dos demais bens e serviços.

Em se tratando de biodiversidade, a questão do valor é ainda mais complexa, porque não existe um pleno conhecimento teórico a respeito desse tema para um gerenciamento adequado destes recursos (FREEMAN, 1979, MOTA, 1997, 2006; PEARCE; TURNER, 1990). Apesar disso, é preciso remediar as deficiências do mercado no que tange ao tratamento dos bens naturais, por exemplo, por meio de instrumentos econômicos e de controle, como multas, taxas e indenizações (FALCIN, 2002).

Atualmente há algumas tentativas metodológicas para colocar em bases econômicas os recursos naturais. Estas técnicas têm o objetivo de tornar explícito o valor econômico do meio ambiente, gerando subsídios para a tomada de decisão. Entretanto é válido salientar que existem desacordos a respeito destas metodologias no que concerne à sua eficiência para cumprir com a finalidade pretendida, implicando que nenhuma delas seja universalmente aceita (MOTTA, 1997; NOGUEIRA; MEDEIROS; ARRUDA, 2000; SANTOS, 2009).

Conforme a literatura econômica, o valor do meio ambiente é composto por quatro parcelas distintas (BENAKOUCHE; CRUZ, 1994; PEARCE; TURNER, 1990):

1. Valor de uso direto: pode ser obtido diretamente pelos preços de mercado, pois relaciona o recurso ambiental com a atividade econômica. São exemplos do uso direto a receita obtida com a pesca, a caça, a venda de madeira, o lazer e o turismo, entre outros;

2. Valor de uso indireto: diz respeito aos benefícios de um maior bem-estar decorrente do equilíbrio das condições naturais do ambiente;

3. Valor de opção: refere-se à decisão de preservar no presente para consumir no futuro, sendo o adiamento do consumo da geração atual em prol das próximas gerações;

4. Valor de existência: influenciado por questões morais, éticas e culturais, e traduzindo o desejo altruísta dos indivíduos em garantir o recurso, pode ser definido a partir do valor intrínseco do bem natural.

1 Preço é a expressão monetária do valor (MANKIW, 2008). 
Existem três metodologias principais para avaliação dos recursos naturais, que aparecem recorrentemente nos trabalhos científicos, e que se dedicam a realizar a valoração ambiental:

1. Método da Valoração Contingente (MVC): para aplicar o MVC é preciso simular o mercado hipoteticamente por meio de questionários. Esses tentam extrair do entrevistado tanto a sua disposição a pagar (DAP) para manter as atuais disponibilidades de recursos naturais como a disposição a receber (DAR) dos indivíduos para aceitar uma diminuição na quantidade de ativos ambientais (HANEMMAN, 1994; AUSTRALIAN GOVERNMENT PUBLISHING SERVICE, 1995).

2. Método do Custo da Viagem (MCV): observa-se a demanda pela atividade recreacional de uma determinada região e os custos em que o visitante incorre para usufruir das amenidades deste recurso (MOTTA, 1997). Por amenidades entende-se o encanto de uma determinada área - portanto um substantivo abstrato - neste trabalho traduzindo uma interseção entre os conceitos de valor de uso indireto e valor de existência.

3. Método de Preços Hedônicos (MPH): comumente utiliza os preços dos imóveis como uma variável proxy do valor dos bens naturais de uma certa localidade. Quanto maior o valor do imóvel, ceteris paribus, maior o valor dos atributos ambientais circundantes ao imóvel considerado (MOTTA, 1997; SOUSA; ÁVILA; SILVA, 2006). Assim como o MCV, para definir o valor dos recursos o $\mathrm{MPH}$ se utiliza dos mercados de bens complementares - respectivamente, os gastos realizados com a viagem até o local em que o recurso se encontra, e o valor dos imóveis.

Contudo estas técnicas, apesar de partirem de suposições válidas para a avaliação do meio ambiente, são insuficientes para capturar todos os aspectos envolvidos no problema da valoração econômica do meio ambiente. Além disso, elas não permitem averiguar todas as parcelas do valor de forma explícita e objetiva, principalmente o valor de uso indireto e o valor de existência. Da mesma forma, falham ao desconsiderar as incertezas associadas tanto a um determinado uso do recurso como à preservação (MOTTA, 1997).

Por último, a irreversibilidade da ação no ambiente também não é contabilizada pelos métodos tradicionais de avaliação. Isso é preocupante, na medida em que, quanto mais reversível for uma ação, menores são os custos ambientais, o que torna o conceito de irreversibilidade importante na avaliação do projeto que irá destruir o meio ambiente (PINDYCK, 1994, 1999; HENRY, 1974; DALY; FARLEY, 2003). Quanto mais irreversível a mudança, maiores serão os danos ao meio, o que torna possível que aumente a propensão da sociedade em optar pela preservação, fato que deve ser computado na avaliação dos custos e dos benefícios da opção de degradação. De acordo com Henry (1974) e com Basili (1997), ao se integrarem no processo de decisão as incertezas e a irreversibilidade das mudanças relacionadas ao uso do bem ambiental, obtém-se um valor adicional decorrente da opção de adiar, denominado de valor de quasi opção.

Assim é que, ignorando-se as parcelas do valor de existência e do valor de uso indireto, assim como as incertezas e a irreversibilidade da ação, o valor gerado para o recurso natural a partir das técnicas convencionais será subestimado (OLIVEIRA, 2005). No caso do MVC, apesar de esta técnica implicitamente apurar o valor de existência, observa-se que não é possível explicitá-la.

Por conseguinte, novos conceitos são necessários na tentativa de suprir algumas das limitações dos métodos atualmente conhecidos. Estas limitações surgem a partir de duas vertentes: 1) das várias incertezas referentes ao uso dos recursos, e 2) devido à irreversibilidade de uma ação no ambiente. No primeiro caso, elas são de diversas naturezas: econômica, ecológica, quanto ao melhor uso do recurso ambiental, nas mudanças tecnológicas, nos benefícios de uso versus benefícios da preservação, e no valor de existência. Neste sentido, a teoria de opções reais fornece conceitos que podem ser de grande valia na tentativa de se obter estimativas mais precisas na valoração econômica do meio ambiente.

Tendo em vista os aspectos que foram mencionados, é possível observar que incertezas, irreversibilidade, valor de existência e valor de uso indireto são lacunas existentes nas técnicas de valoração ambiental que são largamente aplicadas atualmente. Percebe-se que, quanto mais intangível é a parcela do valor considerada, mais difícil é mensurá-la. Diante disto, há necessidade de desenvolver uma nova metodologia fundamentada em conceitos diferentes daqueles provenientes da economia do bem-estar: DAP (disposição a pagar) e mercados complementares e hipotéticos. A isto se propõe o presente estudo. 


\title{
A TEORIA DE OPÇÕES REAIS NO CONTEXTO DE VALORAÇÃO DE RECURSOS NATURAIS
}

De acordo com Pindyck (1999), em projetos de investimentos que envolvem bens naturais há certas peculiaridades envolvidas: 1) a irreversibilidade do investimento, 2) a possibilidade de adiamento da decisão de investir e os benefícios da espera, 3) a escolha do momento ótimo para a utilização do bem ambiental. A presença destes três aspectos sugere que a avaliação destes projetos pelas técnicas que tradicionalmente vem sendo usadas é inconsistente. Neste caso, a abordagem por opções reais permite fazer uma modelagem mais adequada do problema.

Conforme a literatura econômica, o valor do meio ambiente é composto por três parcelas distintas: 1) valor de uso 2) valor de opção 3) valor de existência.

Em síntese:

\author{
Valor Total do Ambiente (VTA) = "valor de uso" (direto + indireto) + "valor de \\ quasi opção" (valor em se adiar o uso dos recursos) + "valor de existência" + \\ "valor de opção"
}

Deve ser ressaltado que, apesar de ser reconhecida na literatura a presença destas três parcelas no valor total do ambiente, grande parte dos trabalhos não estabelecem efetivamente uma análise para as parcelas do "valor de existência" e do "valor de quasi opção". Verifica-se que o objetivo das pesquisas econômicas na avaliação do ambiente tem se prendido fortemente na exploração do valor de uso, seja em termos de benefícios, disposição a pagar, medição da satisfação ou eficiência econômica. No entanto a não consideração explicita do "valor de quasi opção" e do "valor de existência" tende a subestimar a avaliação dos recursos.

Obviamente, declarar valores para as parcelas mencionadas é uma tarefa complexa e desafiadora, principalmente se for considerado que os gostos e as preferências dos indivíduos e o valor das espécies (vegetação e fauna) modificam-se no decorrer dos anos, ou seja, são variáveis que evoluem estocasticamente no tempo. Isso traz uma complexidade maior ao problema. Neste contexto, a teoria das opções reais permite incorporar a aleatoriedade das variáveis de modo endógeno à avaliação do "valor" do recurso num ambiente em que as decisões têm algum grau de irreversibilidade associado.

Sucintamente falando sobre a teoria de opções reais, este método vem ganhando espaço crescente nas mais diversas áreas de pesquisas e se constitui num instrumento complementar às técnicas tradicionais de análise de investimentos (especialmente o VPL - valor presente líquido e a TIR - taxa interna de retorno), em diversos setores da economia (DIXIT; PINDYCK, 1994).

A teoria das opções reais (OR) tem sido definida como a moderna ferramenta para análise de decisões de investimentos considerando a natureza estocástica das variáveis de incerteza do problema. Embora atualmente sua aplicação seja mais frequente na área de finanças, o conceito de opção foi inicialmente usado em aplicações de economia ambiental, enfatizando a regra de decisão ótima em investimentos que são total ou parcialmente irreversíveis. A irreversibilidade aparece no debate da valoração dos recursos naturais associada à noção de que existe um custo extra no uso do meio ambiente, que está além do desembolso com o investimento inicial do projeto. Este custo refere-se à perda total ou parcial do "valor de existência" e do "valor de opção".

No contexto das opções reais, a avaliação dos recursos naturais e de projetos de investimento que os envolve considera alguns importantes aspectos (HENRY, 1974; INSLEY, 2002):

- irreversibilidade;

- timing;

- incertezas;

- flexibilidade gerencial. 
A irreversibilidade da mudança em caso de investimentos com ativos ambientais relaciona-se não apenas com os custos financeiros da decisão de investir, já que o dinheiro gasto na maioria das vezes é um custo afundado e ao menos parcialmente irrecuperável. Mas, sobretudo, deve ser também considerado que a decisão de realizar qualquer investimento no meio ambiente implica modificar o seu estado natural e quase sempre causar prejuízos ambientais. Essa mudança pode ser assumida como sendo irreversível, dependendo do tempo que a natureza levará para se recuperar dos danos sofridos. Portanto a decisão, em termos de um ativo ambiental, significa um comprometimento dos recursos naturais, além dos recursos financeiros. A irreversibilidade pode ser total ou parcial (PINDYCK, 1999).

Quanto ao timing, esse termo tem sido empregado para designar o momento ótimo de se realizar o investimento. Raramente as decisões são do tipo "agora ou nunca" e esperar para investir é vantajoso até o instante em que a decisão de investir, que depende de variáveis com incerteza, alcance um determinado valor chamado "valor de gatilho" (instante em que o investimento imediato é a melhor decisão). Assim, existe um prêmio na espera até que as variáveis incertas alcance o seu "gatilho". A variável, quando está no gatilho, normalmente é destacada pelo sinal de asterisco (*) (PINDYCK, 1991; PINDYCK, 2001).

A expressão timing designa a espera para usar os recursos naturais até encontrar o momento em que o prêmio da espera torna-se nulo. Assim, deve-se comparar o custo de adiamento com os benefícios provenientes do adiamento da decisão para determinar o " $t$ " ótimo do investimento.

Em se tratando das incertezas, existem incertezas diversas relacionadas aos bens naturais:

- Incerteza econômica

- Incerteza ecológica

- Incerteza quanto ao melhor uso do recurso ambiental

- Incertezas nas mudanças tecnológicas

- Redução da incerteza pela revelação da informação

- Incertezas nos benefícios de uso versus benefícios da preservação

- Incerteza no "valor de existência".

Sobre os dois primeiros tipos de incertezas apontadas e seus desdobramentos:

1) Incerteza econômica: refere-se ao fato dos impactos de um investimento ou de uma política ambiental serem incertos. Não se pode garantir como a agricultura, a pesca, o turismo e a rotina de vida da população serão afetados pelo projeto realizado.

2) Incerteza ecológica: existe incerteza na forma como o ambiente (fauna, vegetação, ar, temperatura, acidez das águas e solo) será impactado. Aí se incluem também as incertezas quanto ao tempo de recuperação da área afetada.

Quanto às demais incertezas, apontam-se as seguintes observações:

3) Normalmente são ignoradas as melhores escolhas de uso para o recurso natural que estão relacionadas com as circunstâncias do momento.

Mudanças tecnológicas podem acontecer e, por exemplo, reduzir o tempo de recuperação do meio ambiente a uma degradação sofrida.

Quando se decide pela degradação e pelo uso dos recursos naturais de uma determinada área, se um projeto de uso alternativo anterior já foi feito em outra área com características semelhantes, a incerteza diminui no próximo investimento devido à revelação da informação acontecida com o primeiro projeto. A chegada de novas informações reduz a incerteza em investimentos sequenciais.

Há incertezas tanto nos benefícios de se utilizar o bem ambiental quanto nos benefícios da preservação.

4) Incertezas existem também no próprio "valor de existência" do recurso, se for considerado que este valor tem uma relação com aspectos morais e culturais que se modificam no decorrer dos anos. 
Quanto ao conceito de flexibilidade gerencial, este significa considerar formalmente na modelagem um aspecto que é bastante intuitivo na prática: as decisões estratégicas são revisadas ao longo do tempo se as condições iniciais em que o projeto foi avaliado se alteram.

Em suma, todos estes aspectos destacados nessa seção são desconsiderados pelas técnicas que atualmente estão sendo usadas para valorar o meio ambiente. Mas com a teoria de opções reais há condições de introduzi-los explicitamente na modelagem. Ressalta-se que avaliar o meio ambiente e conhecer o seu valor é um aspecto essencial para se avaliar usos alternativos ao estado de conservação.

\section{METODOLOGIA}

A metodologia descrita nesta seção é inédita na literatura e sua aplicação se deu a partir de dados sintéticos que permitem ilustrar como o método pode ser aplicado em situações reais. Assim, para se alcançar o objetivo deste trabalho, a pesquisa assumiu algumas suposições como as colocadas a seguir:

- A valoração do meio ambiente foi feita por meio do cálculo explícito das parcelas do valor de uso indireto e do valor de existência, de forma a evitar-se a subestimação do valor dos recursos naturais;

- Estas parcelas compõem o valor total do meio ambiente, implicando que ignorá-las significa subestimar o valor dos recursos naturais, podendo gerar distorções no processo de tomada de decisão;

- Valor de uso direto foi entendido como os bens, os serviços e as atividades provenientes da natureza e que servem para o consumo dos indivíduos. Como esta parcela do valor possui preços de mercado - por exemplo, pesca, caça, madeira, navegação e turismo, que são bens e serviços que podem ser transacionados nos mercados convencionais - seu valor pode ser computado mais facilmente.

- Para a análise do valor de uso direto foram considerados a receita turística e dois produtos: o milho e a soja. O raciocínio empregado para avaliar o valor de uso direto considerando estes dois produtos pode se estender para qualquer quantidade e variedade de bens.

- As séries de preços e de quantidades - mensais e referidos ao período de janeiro de 2000 a maio de 2009 - para ambos as commodities foram obtidas na Associação Brasileira das Indústrias de Óleos Vegetais (ABIOVE, 2010).

- A análise do valor da atividade turística foi realizada a partir da série de gastos dos turistas na Bahia, construída sinteticamente por interpolação linear a partir dos dados da Bahiatursa (órgão oficial de turismo do estado da Bahia/Brasil) que constam no relatório da Câmara Americana Bahia (BAHIATURSA, 2005).

- Para estimar os parâmetros de drift e de volatilidade do processo estocástico da série dos produtos agrícolas e das receitas turísticas foram usados dados mensais.

- A série de dados de fluxo de receita foi criada sinteticamente.

Para a série de número de visitantes $(R S)$ foram utilizados dados anuais informados pela Bahiatursa (BAHIATURSA, 2010).

Valor de uso direto foi calculado como função dos preços e das quantidades das variáveis consideradas e da receita turística, conforme a equação (1):

$$
\text { Valor_de_Uso_Direto }=f(\text { preços, quantidades, receitas })
$$

Por último, cumpre informar que o valor de uso direto incorpora as incertezas por meio de um processo estocástico apropriado. Supõe-se que, assim como a receita turística, os preços e as quantidades destes produtos seguem um Movimento Geométrico Browniano (MGB). 
O MGB é um processo estocástico plausível para séries de preços, pois possui uma distribuição log-normal de forma que o preço não poderá ser um valor negativo. Como particularidades, tem-se que o MGB é o processo estocástico mais popular, sendo um processo de Marco. No processo MGB, quanto maior o horizonte de previsão, maior a volatilidade. Pode existir uma tendência exponencial de crescimento ou de queda, a variância cresce com o tempo, os incrementos são independentes e estacionários, e os preços (ou variável de incerteza) possuem distribuição log-normal.

A equação (2) reporta à equação do MGB, onde $\boldsymbol{H}$ é a tendência do processo, $\sigma$ é a variância e $d z$ é o incremento do processo de Wiener em que $d z=\varepsilon_{t} \sqrt{t}$ e em que ${ }^{\varepsilon_{\mathrm{r}} \text { é }} N\left(\varepsilon_{t} \varepsilon_{s}\right)=0$ para $t \neq s$ :

$$
d P=\mu P d t+\sigma P d z
$$

O valor esperado do projeto (V) em (t) é dado pela expressão (3):

$$
E[V(t)]=V_{0} e^{\alpha t}
$$

Onde $V_{\mathrm{o}}$ é o valor inicial do projeto.

A variância do processo MGB é dada pela expressão (4):

$$
\operatorname{Var}[V(t)]=V_{0}^{2} e^{2 \alpha t}\left(e^{\sigma 2}-1\right)
$$

A equação geral do modelo MGB é dada pela expressão (5),

$$
d V=\alpha V d t+\sigma V d z
$$

Onde $\alpha$ é a tendência do processo, e $\sigma$ é a variância do processo.

A Figura 1 esboça um processo simulado do MGB, permitindo notar algumas das características descritas e que a variância cresce com o tempo.

Figura. 1. Movimento Geométrico Browniano simulado

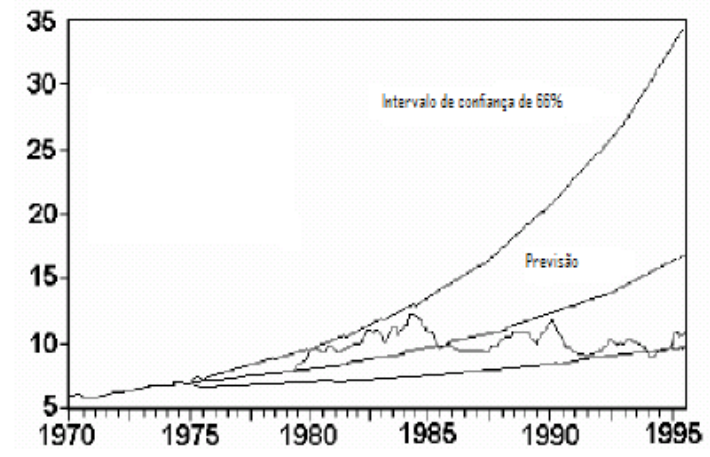

Fonte: in Dixit e Pindyck (1994)

\section{RESULTADOS DA PESQUISA}

Feita a suposição de que o processo que descreve as três séries (milho, soja, receita turística) é um MGB, os parâmetros foram obtidos conforme apresentado por Gonçalves e Ferreira (2008). As tendências das séries de preços, de quantidades e de receita turística foram calculadas como mostrado respectivamente nas equações (6), (7) e (8). 


$$
\begin{aligned}
& d r i f t=\text { Média }\left(\ln \frac{P_{t+1}}{P_{t}}\right) \\
& d r i f t=M e ́ d i a\left(\ln \frac{Q_{t+1}}{Q_{t}}\right) \\
& d r i f t=M e ́ d i a\left(\ln \frac{\theta_{t+1}}{\theta_{t}}\right)
\end{aligned}
$$

A volatilidade foi estimada por meio de um estimador não enviesado para o desvio-padrão da amostra como mostra a equação (9):

$$
\sigma=\sqrt{\frac{\sum_{i=1}^{n}\left(u_{i}-\bar{u}\right)^{2}}{n-1}}
$$

Onde:

$$
\sigma=\ln \frac{P_{t}}{P_{t-1}} \quad u=\ln \frac{\theta_{t}}{\theta_{t-1}}, \mathrm{e}^{\bar{u}}=\text { drift= tendência }
$$

As equações do Movimento Geométrico Browniano das séries utilizadas estão representadas pelas equações (10) a (14):

$$
\begin{aligned}
& \frac{d P_{\text {milho }}}{P}=0,015 d t+0,07 d z \\
& \frac{d Q_{\text {milho }}}{Q}=0,04 d t+0,21 d z \\
& \frac{d Q_{\text {soja }}}{Q}=0,04 d t+0,62 d z \\
& \frac{d P_{\text {soja }}}{P}=0,05 d t+0,151 d z \\
& \frac{d \theta}{\theta}=0,04 d t+0,08 d z
\end{aligned}
$$

Na modelagem das quantidades e dos preços da soja e do milho e das receitas turísticas, considerou-se que os parâmetros descrevem uma taxa mensal:

$$
\begin{aligned}
a & =\% \text { a.m } \\
\sigma & =\% \text { a.m }
\end{aligned}
$$

\section{Valor de uso direto}

Para o valor de uso direto, a Figura 2 apresenta alguns dos possíveis caminhos simulados para a série de preço da soja. O objetivo da simulação por MGB é incorporar as incertezas futuras no valor atual da parcela "valor de uso direto". Estão mostradas a simulação real (linha superior) e a simulação neutra ao risco (linha inferior). Apesar de terem sido feitas as simulações reais e neutra ao risco ${ }^{1}$, neste trabalho apenas a série simulada com tendência neutra ao risco foi utilizada, exceto para a série de quantidades. A Figura 3 reporta alguns dos possíveis caminhos simulados para a 
série de quantidades produzidas de soja.

Destaca-se que, uma vez feita a simulação dos preços, é preciso trazer os valores ao tempo presente por um fator de desconto.

Figura 2: Simulação dos preços da soja

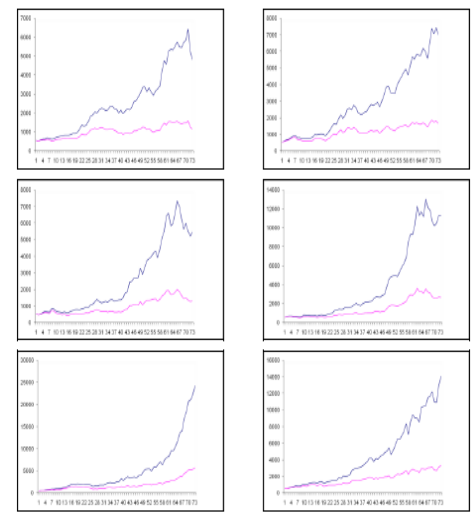

Figura 3: Simulação das quantidades de soja

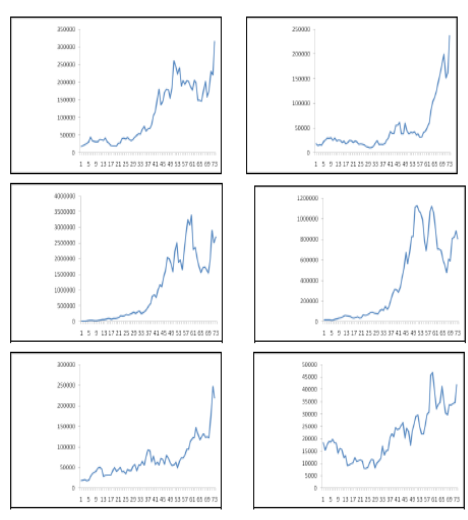

\section{Valor de uso indireto}

O valor de uso indireto foi avaliado como os benefícios relacionados ao lazer, à recreação e ao bem-estar que um determinado bem ambiental proporciona aos seus visitantes. Dentre outros, este benefício se relaciona indiretamente com a manutenção da diversidade genética, com a provisão de recursos básicos (oxigênio e água) e com a proteção dos corpos d'água. Isto é, quanto melhor as condições naturais do ambiente, maior o bem-estar que ele proporcionará à sociedade. Portanto a parcela "valor de uso indireto" é intangível e, por isso, mais difícil de mensurar.

A modelagem do valor de uso indireto seguiu o raciocínio apresentado por Conrad (1997). Para tanto, se recorreu a uma variável denominada de amenidade $(A)$, observada por meio da proxy número de turistas. Segundo Otto, Monchuk, Jintanakui e Kling (2007), a amenidade de uma região relata a qualidade dos recursos naturais ali existentes. A partir da definição feita por estes autores, esta pesquisa recorreu à variável proxy fluxo de turistas para relacionar a perda dos benefícios indiretos dos recursos naturais com uma diminuição na amenidade da área, o que deverá acarretar uma retração do fluxo de turistas.

A amenidade, no estudo aqui proposto, pode ser entendida como atributos que tornam agradável a vida das pessoas em certo lugar. Se a amenidade de uma região diminui por uma perda de capital natural, o fluxo turístico se reduz, assim como a receita turística. Logo, nesta pesquisa a amenidade foi utilizada como forma de se obter uma estimativa para o valor de uso indireto que está relacionado com a qualidade dos recursos naturais e com o equilíbrio da natureza.

A variável amenidade é calculada como sendo proporcional à taxa de visitação. Desta forma, a partir da série fluxo de turistas, pode-se fazer evoluir a variável amenidade por meio de um processo estocástico MGB. Observe-se que a série de visitação em certa região pode ser computada de maneira mais simples que a variável amenidade, cujos atributos são abstratos.

Na expressão (16) a amenidade é colocada como proporcional à taxa de visitação, e na expressão (17) faz-se a amenidade evoluir segundo os parâmetros da série de visitação (Rs). O processo descrito na expressão (17) para a amenidade evolui seguindo a trajetória apresentada na Figura 4. 
Figura 4: Amenidade evoluindo ao longo do tempo

$$
\begin{aligned}
& A=\eta R S \quad d A=\mu A d t+\sigma A d z \\
& \text { A: amenidade } \quad \text { A: amenidade } \\
& \eta \text { : constante de proporcionalidade } \quad \mu \text { : parâmetro de drift da série de amenidade } \\
& \text { Rs: taxa de visitação } \quad \sigma \text { : volatilidade da série de amenidade } \\
& \begin{array}{l}
A^{*}=\frac{[\alpha(\delta-\mu)]}{\alpha+1} \mathrm{~N} \\
-\alpha=(-\beta)=\left(\frac{1}{2}-\frac{\mu}{\sigma^{2}}\right)-\sqrt{\left(\frac{1}{2}-\frac{\mu}{\sigma^{2}}\right)^{2}+\frac{2 \delta}{\sigma^{2}}} \quad \begin{array}{l}
\text { \%: retorno sem risco } \\
\text { it taxa de crescimento da amenidade. }
\end{array}
\end{array}
\end{aligned}
$$

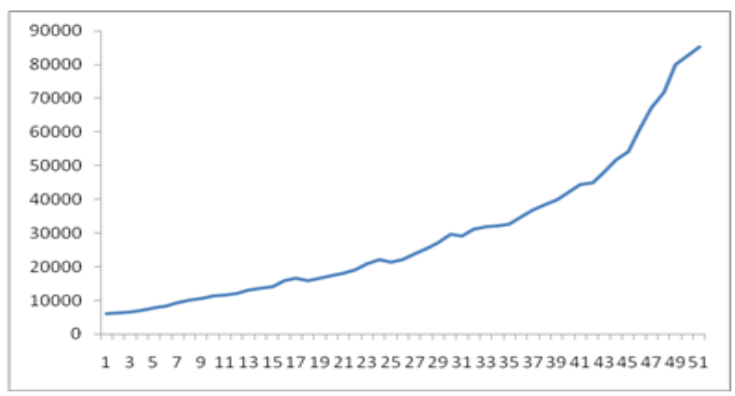

Figura 7.12: evolução estocástica do valor existência

\section{Valor de existência}

A parcela do valor de existência é calculada relacionando-a com o valor de uso indireto e de amenidade. Considerou-se que o valor de existência - que traduz o valor intrínseco que os indivíduos atribuem à preservação das espécies - pode ser descrito em duas partes, conforme mostrado na equação (18): efeitos morais, éticos, culturais e psicológicos proporcionados pelos recursos naturais (representados por k), e amenidade ou encantamento dos recursos naturais (representada por $A$ ).

$$
\text { Valor de existência }=k+\text { amenidade }(A)
$$

A variável $(k)$ pode ser avaliada a partir de questionários, como propõe a tradicional metodologia de valoração contingente, buscando extrair a disposição a pagar dos indivíduos (DAP) para preservar os recursos naturais. Vale lembrar aqui que a DAP é a disposição média a pagar multiplicada pelo número de indivíduos da população da qual a amostra entrevistada foi retirada.

Uma vez aplicado o questionário, pode-se apurar a DAP no instante de tempo presente. Com a DAP em T $=0$, deixa-se a DAP evoluir estocasticamente conforme os parâmetros drift e a variância da variável amenidade calculada conforme o procedimento mostrado para se avaliar a parcela valor de uso indireto. Assim, DAP $=\eta$ A (observe que $\eta$ faz a DAP proporcional a amenidade).

Logo, se A aumenta, a DAP aumenta. Se A diminui, a DAP diminui. Desta forma, relaciona-se a DAP em $\mathrm{t}=0 \mathrm{com}$ os valores futuros da amenidade para estimar os valores da DAP nos períodos $(t+k)$, com $k=1,2,3 \ldots$ Ressalva-se que a DAP obtida em $\mathrm{T}=0$ é determinística.

Portanto:

1) $D A P(t=0) \rightarrow$ obtida, significando que DAP em $t=0$ é avaliada por meio de questionários e de entrevistas

2) $\triangle \% D A P(t+k) \rightarrow(\triangle \%$ Amenidade $)$, significando que a variação da DAP nos períodos seguintes àquele obtido inicialmente pelo questionário será proporcional à variação na variável amenidade.

3) $\triangle \% D A P(t+k)$ representa o valor intrínseco da existência evoluindo estocasticamente no tempo. 
Este trabalho sugeriu uma metodologia para modelar endogenamente e explicitamente as parcelas "Valor de Uso Direto", "Valor de Uso Indireto", "Valor de Existência", necessárias na definição do valor econômico do meio ambiente, a partir das incertezas no valor futuro dos recursos naturais e no seu uso.

Um dos pontos relevantes da metodologia apresentada foi a possibilidade de explicitar as parcelas que compõem o valor total do meio ambiente. Como discutido nas primeiras seções, as principais técnicas de valoração atualmente existentes não visam calcular todas as parcelas especificadas na definição do valor econômico dos recursos naturais. Logo o valor obtido pelas mesmas tende a ser subestimado.

O valor econômico do meio ambiente, em determinado instante de tempo, foi obtido pelo valor presente e futuro das atividades econômicas selecionadas no exemplo para a modelagem na região - soja e milho e turismo - (valor de uso direto), pelo valor de uso indireto (mínimo de amenidade que justifica a preservação), assim como pelo valor de existência presente e futuro da região.

A inclusão do valor futuro na determinação do valor presente dos recursos naturais é imprescindível para que os órgãos competentes, autoridades e a sociedade conheçam, a cada instante de tempo, se é mais vantajoso conservar ou degradar o meio ambiente, a fim de dar continuidade ao processo do desenvolvimento humano. Desta forma, espera-se reduzir o impacto negativo do processo de desenvolvimento sobre o bem-estar social e o trade-off que naturalmente foi criado entre crescimento versus ecossistema.

\section{REFERÊNCIAS}

ABIOVE (2010) - Brazilian Association of Vegetable Oil Industries - (www.abiove.com.br).

AUSTRALIAN GOVERNMENT PUBLISHING SERVICE. Techniques to Value Environmental Resources: an Introdutory Handbook. 1995. Disponível em: http://www.environment.gov.au/about/publications/ economics/value/chapter2.

BAHIATURSA - Secretaria de Turismo da Bahia - (www.bahiatursa.ba.gov.br/site/).

BASILI, M. Quasi-Option Value and Hard Uncertainty. Quaderni del Dipartimento di Economia Política. Universita' degli Studi di Siena. N²16. Outubro de 1997.

BENAKOUCHE, R.; CRUZ, R.S. Avaliação monetária do meio ambiente. São Paulo: Ed. Makron Books, 1994.

BRAGA, P.L.S.; OLIVEIRA, C.R. Valoração econômica do Parque Nacional da Lagoa do Peixe, RS. 2003. Disponível em: www.ich.ufpel.edu.br/economia/professores/xavier/PNLP_DAP_SOBER2005. pdf).

CONRAD, J. M. Resource Economics. Cambridge University Press. 1999.

DARLY, H \& FARLEY, J. Ecological Economics Principles and Aplications. 2003.

DIXIT, A. \& PINDYCK, R. Investment Under Uncertainty. Princeton: Princeton University Press, 1994.

EUTÁCHIO, J. A. V; TÁVORA, J. L. T. Metodologias de avaliação de ativos ambientais: uma comparação entre as medidas. III Encontro Nacional da sociedade brasileira de economia ecológico. 1999.

FACIN, M. A. Meio Ambiente e Direitos Humanos. 2002. Disponível em: http://jus2.uol.com.br/Doutrina/texto.asp?id=3463. Acesso em: 2010.

FARIA, H. M; SILVA, R. J. Oportunidades Econômicas com a Gestão Ambiental. Escola Federal de Engenharia de Itajubá. Revista Pesquisa e desenvolvimento tecnológico. 23 (3)- 153-162. Itajubá, 1999.

FREEMAN, A. M. The Benefits of Environmental Improvements. Theory and Practice, The John Hopkins University Press, Baltimore, 1979. 
HILDEBRAND, E. et al. "Valoração Contingente" na avaliação econômica de áreas verdes urbanas. Revista Floresta vol. 32, 1. ed. (121-132). Parana, 2002.

HENRY, C. Investiment Decisions Under Uncertainty: The "Irreversibility effect". The American Economic Review. Vol. 64, nº 6, p 1006-1012. 1974.

INSLEY, M. A Real Options Approach to the Valuation of a Forestry Investment. Journal of Environmental Economics and Management. Vol. 44, p. 471-492. 2002.

OLIVEIRA, A. O. P. Operação independente por subsistemas: comportamento estratégico para a geração no sistema elétrico brasileiro. UFRJ/Coppe (tese de doutorado). 2005

MANKIW, N. G. Introdução à Economia: Tradução da 3a Edição Norte-Americana. São Paulo: Editora Pioneira Thomson Learning, 2008.

MATTOS, K. M. C. Valoração ambiental no processo produtivo. Congresso em Engenharia de Produção. 1998. Disponível em: http://www.abepro.org.br/biblioteca/ENEGEP1998_ART500.pdf).

MOTA, J. A. O valor da Natureza: Economia e política dos recursos naturais. Rio de Janeiro. Ed. Garamond. 2006.

MOTA, J. A. O valor da Natureza: Economia e política dos recursos naturais. Rio de Janeiro. Ed. Garamond. 2006

MOTTA, R. S. Manual para Valoração Econômica dos Recursos Naturais. Ministério do Meio Ambiente, dos Recursos Hídricos e da Amazônia Legal. 1997.

MOTTA, R. S. Desafios ambientais da economia brasileira. Texto para discussão No 509 . IPEA. 1997a.

MOTTA, R.S. $O$ uso de instrumentos econômicos na gestão ambiental. 2000. Disponível em: http:// www.undp.org.cu/eventos/instruverdes/Instr\%20Econ\%20Gestion \%20Ambiental\% 20R\%20Seroa\%20 da\%20Motta.pdf. Acesso em: 2009.

NOGUEIRA, J.M; MEDEIROS, M. A. A; ARRUDA, F.S.T. et. al. Valoração Econômica do meio ambiente: ciência ou empirismo? Cadernos de Ciência \& Tecnologia. Brasília. Volume 17, n², p 81-115, maio/ ago. 2000.

OLIVEIRA, J.A. Estimativa do valor econômico do recurso ambiental do ar atmosférico - VERAar - da frota a diesel do sistema integrado de transporte de fortalece- SIT-FOR. Anais do XV congresso Brasileiro de transporte e trânsito. 2005.

OTTO, D.; MONCHUK, D. ET AL. The Economic Value of Iowa's Natural Resources. Iowa state University. Dezembro de 2007.

PEARCE,D.; TURNER, R. Economics of natural resources and the environment. Baltimore: The Johns Hopkins University Press, 1990.

PINDYCK, R. S. Irreversibilities and the timing of environmental policy. Massachusetts Institute of technology, Cambridge, 1999.

PINDYCK, R. S. Optimal timing problems in environmental economics. Massachusetts Institute of technology, Cambridge. 2001.

PINDYCK, R.S. Irreversibility, Uncertainty and Investiment. Journal of Economic Literature. Vol 29, p. $1110-1152.1991$.

SANTOS, R.B. Relações entre o meio ambiente e ciência econômica: reflexões sobre economia ambiental e a sustentabilidade. Disponível em: http://www.fae.edu/publicacoes/ pdf/IIseminario/ pdf_reflexoes/reflexoes_23.pdf. Acesso em: 2009.

SOUZA, A. ; ÁVILA, S. C. ; SILVA, W.V. Modelos de preços hedônicos para estimar a relação preço-satisfação na compra de veículos populares novos. Revista Economia \& Gestão. Vol 07, n 15. 2006.

SOUZA, R. F. P. Economia do meio ambiente e responsabilidade social: os métodos de valoração econômica e controle ambiental. XLV Congresso da Sociedade Brasileira de Economia, Administração e Sociologia Rural. Londrina, 2007. 
SOUZA, R. F. P; SOUZA. M. F. P. Políticas ambientais e a determinação da escala sustentável de utilização dos recursos naturais. XLVI Congresso da Sociedade Brasileira de Economia, Administração e Sociologia Rural. Acre, 2008.

\section{NOTA}

Tendência neutra ao risco é um artifício matemático que penaliza a tendência real do processo para que se possa utilizar a taxa de desconto livre de risco. 